\title{
Nonneutralized charge effects on tokamak edge magnetohydrodynamic stability
}

\author{
Linjin Zheng, ${ }^{1)}$ W. Horton, ${ }^{1)}$ and H. Miura, ${ }^{2)}$ T. H. Shi, ${ }^{3)}$ and H. Q. Wang ${ }^{3)}$ \\ 1) Institute for Fusion Studies, University of Texas at Austin, Austin, TX 78712 \\ 2) National Institute of Natural Sciences, \\ 322-6 Oroshi, Toki, Gifu 509-5292, Japan \\ 3) Institute of Plasma Physics, Chinese Academy of Sciences, Hefei, Anhui, China
}

(Dated: March. 27, 2016)

\begin{abstract}
Owing to the large ion orbits, excessive electrons can accumulate at tokamak edge. We find that the nonneutralized electrons at tokamak edge can contribute an electric compressive stress in the direction parallel to magnetic field by their mutual repulsive force. By extending the ChewGoldburger-Low theory [Proc. R. Soc. London, Series A 236, 7 (1956)], it is shown that this newly recognized compressive stress can significantly change the plasma average magnetic well, so that a stabilization of magnetohydrodynamic modes in the pedestal can result. This linear stability regime helps to explain why in certain parameter regimes the tokamak high confinement can be rather quiet as observed experimentally.
\end{abstract}

PACS numbers: 52.53.Py, 52.55.Fa, 52.55.Hc 
Tokamak edge stability is important for achieving/maintaining the so-called high confinement (H-mode) [1]. It was shown earlier that a self-consistent radial electric sheath can develop at tokamak edge $[2,3]$. Experiments show that an inward radial electric field at edge can significantly improve tokamak confinement, as reviewed in Ref. 4.

The conventional hypothesis is that the transport barrier is formed as a result of the suppression of microturbulence by the $\mathbf{E} \times \mathbf{B}$ flow shear, where $\mathbf{E}$ and $\mathbf{B}$ represent electric and magnetic fields $[5,6]$. The rotation decorrelation of turbulence eddies is further shown to rely on the reduction of local magnetic shear [7], resulting from the bootstrap current in the pedestal. However, experimental observations show that, before the so-called edge localized modes (ELMs) develop, there is a discharge period during which the H-mode confinement is rather quiet (see the $\mathrm{H}$ mode periodsin Fig. 15 in Ref. [8]). This indicates that certain parameter regimes in H-mode confinement are actually linearly stable. The ELM excitation occurs only after the density and temperature profiles evolve further.

Furthermore, the numerical equilibrium calculations in Ref. 9 show that the bootstrap current in the pedestal can cause a significant reduction of local magnetic shear. Weaker magnetic shear in the pedestal leads the plasma to become unstable to the magnetohydrodynamic (MHD) modes [10]. Therefore, the quiet feature of H-mode confinement as observed in C-Mod [8] requires a physical explanation.

In this letter we point out that the nonneutralized electrons at the tokamak edge can contribute an electric compressive stress in the direction parallel to magnetic field. This newly recognized compressive stress can significantly change the plasma average magnetic well, so that a stabilization of pedestal plasma can result. This linear stabilization regime therefore helps to explain the quiet feature of tokamak H-mode confinement. The nonneutral charge effects on electrostatic modes were investigated in Ref. [11]. In this work we focus on the MHD modes.

We first use Fig. 1 to explain the physics. In this figure the small circles depict excessive electrons. As pointed out in Ref. 2, due to the ion orbit losses excessive electrons can accumulate at tokamak plasma edge. As also pointed out in Ref. 3, the edge charge accumulation relies not only on the ion orbit losses, but also on a compensation by the incoming collisional ions. However, the compensation does not fully annul the net ion losses. The building up of radial electric field as observed experimentally indicates that there is indeed accumulation of excessive electrons at plasma edge, as governed by Poisson's equation. Generally, the charge 
density increases from core plasma toward plasma edge. However, the excessive electrons in the scrape-off layer (SOL) tend to escape to divertors and consequently the charge density decreases abruptly across the last closed flux surface. The excessive electron accumulation can induce equilibrium electric field perpendicular to magnetic field [12]. The perpendicular electric field mainly leads to the so called $\mathbf{E} \times \mathbf{B}$ particle drift. Although there is no equilibrium electric field in the parallel direction, the excessive electrons indeed push each other in the parallel direction by their mutual repulsive force. This gives rise to a parallel electric compressive stress (referred to as the e-stress). The e-stress resembles the thermal pressure force: one by the electric repulsive force and the other by the mechanical force. It is because of the parallel e-stress that the excessive electrons are pushed away each other into divertors in SOL. This can also be viewed from the energy picture. For a nonneutral plasma the total energy is the sum of kinetic and electric potential energies. This leads the equation of state change. On the closed magnetic surfaces, however, the magnetic field can hold excessive electrons. Consequently, the repulsion among the excessive electrons generate the parallel e-stress. The more excessive electrons the stronger the parallel e-stress. Since the e-stress has similar nature to plasma pressure, the radial variation of e-stress can contribute to the perturbed energy balance when the interchange type of modes develop. As the e-stress increases outwardly in the pedestal region, one can expect it to contribute an equivalent favorable average magnetic well and consequently stabilize the interchange type of modes.

We extend the Chew-Goldburger-Low (CGL) theory in Ref. 13 to study the e-stress effects. The Vlasov equation is introduced for this purpose:

$$
\frac{\partial f_{j}}{\partial t}+\mathbf{v} \cdot \nabla_{x} f_{j}+\frac{e_{j}}{m_{j}}(\mathbf{E}+\mathbf{v} \times \mathbf{B}) \cdot \nabla_{v} f_{j}=0
$$

where $f$ is distribution function, $j=i, e$ is species index, $e_{j}$ is particle charge, and $m_{j}$ is mass. Following Ref. 13, the $e_{j} / m_{j}$ expansion is introduced, such that $f=f_{0}+\epsilon f_{1}+\cdots$. We also introduce the frame transform: $\mathbf{v}=\mathbf{u}_{0}+\mathbf{v}^{\prime}$, with $\mathbf{u}_{0}=\mathbf{u}_{0 \|}+\mathbf{u}_{E}+\mathbf{u}_{\varphi}, \mathbf{u}_{E}=\mathbf{E} \times \mathbf{B} / B^{2}$, and $\mathbf{u}_{\varphi}$ being determined later. Here and later on, the subscripts $\perp$ and $\|$ are introduced to denote the perpendicular and parallel directions to magnetic field.

The lowest order equation reads

$$
\mathbf{v}^{\prime} \times \mathbf{B} \cdot \nabla_{v} f_{0 j}=0
$$


Here, it has been assumed that $\left(\mathbf{E}_{\|}+\mathbf{u}_{\varphi} \times \mathbf{B}\right) \cdot \nabla_{v} f_{0 j} \sim \epsilon$. The next order equation then becomes

$$
m_{j} \frac{\partial f_{0 j}}{\partial t}+m_{j} \mathbf{v} \cdot \nabla_{x} f_{0 j}+e_{j} \mathbf{v}^{\prime} \times \mathbf{B} \cdot \nabla_{v} f_{1 j}+e_{j}\left(\mathbf{E}_{\|}+\mathbf{u}_{\varphi} \times \mathbf{B}\right) \cdot \nabla_{v} f_{0 j}=0 .
$$

In difference from the CGL theory we have kept the $\mathbf{E}_{\|}$effects, since it contains the e-stress effects. As shown later in Eq. (5) the parallel force balance requires the balance between thermal pressure and electric potential. One cannot require both the parallel pressure gradient and parallel electric field to vanish individually. We also note that, since ion and electron temperatures $T_{j}$ are of same order, the electron equation needs also to be included.

The solution of lowest order equation is $f_{0 j}=f_{0 j}\left(\left(\mathbf{v}-\mathbf{u}_{0 \perp}\right)^{2}, \mathbf{B} \cdot \mathbf{v}, \mathbf{x}, t\right)$. With this solution the ion and electron momentum equations can be constructed and the single fluid equation thus becomes

$$
\rho \frac{d \mathbf{u}_{0}}{d t}=-\nabla \cdot \mathbf{P}+\mathbf{j} \times \mathbf{B}+\nu n_{0} e\left(\mathbf{E}_{\|}+\mathbf{u}_{\varphi} \times \mathbf{B}\right)
$$

where $n_{0}=n_{i}$ denotes ion density, $\nu=n_{e} / n_{0}-1$ describe the charge, $\rho$ is mass density, electron inertia is neglected, $e=e_{i}, \mathcal{P}=p_{\perp}(\mathcal{I}-\mathbf{b b})+p_{\|} \mathbf{b b}$ is the pressure tensor, with $\mathbf{b}=\mathbf{B} / B$ and $\mathcal{I}$ being unit dyadic, and

$$
\begin{aligned}
\frac{d}{d t} & =\frac{\partial}{\partial t}+\mathbf{u}_{0} \cdot \nabla_{x}, \\
n_{0} & =\int d^{3} v^{\prime} f_{0 i}, \\
n_{0} \mathbf{u}_{0} & =\int d^{3} v^{\prime} \mathbf{v} f_{0 i}, \\
\mathbf{j} & =\sum_{i, e} e \int d^{3} v^{\prime} \mathbf{v}^{\prime} f_{1 j}=\frac{1}{\mu_{0}} \nabla \times \mathbf{B} .
\end{aligned}
$$

Here, $\mu_{0}$ is magnetic constant.

In the low frequency regime the parallel projection of Eq. (4) becomes

$$
\mathbf{b} \cdot \nabla P_{\perp}-\mathbf{B} \cdot \nabla \frac{p_{\perp}}{B}+\mathbf{B} \cdot \nabla \frac{p_{\|}}{B}+\nu n e \mathbf{b b} \cdot \nabla \varphi=0
$$

where $p_{\perp, \|}=n_{0} T_{\perp, \|}$, with the temperature $T_{\perp, \|}$ and the relative charge density parameter $\nu$ assumed to be functions of magnetic surface $\psi$. Equation (5) can be solved

$$
n_{0}=\bar{n}\left(\frac{B}{B_{0}}\right)^{1-T_{\perp} / T_{\|}} \exp \left\{\frac{\nu e \varphi}{T_{\|}}\right\},
$$


where $\bar{n}$ is a function of $\psi$ and $B_{0}$ is the normalization constant for magnetic field. In the limit of neutral plasama $\nu=0$ and Eq. (6) reduces to the result by the CGL theory. The pressure can be then expressed as $p_{\perp, \|}=p_{\perp, \|}^{T}+p_{\perp, \|}^{\varphi}$, with

$$
\begin{aligned}
& p_{\perp, \|}^{T}=\bar{n}\left(\frac{B}{B_{0}}\right)^{1-T_{\perp} / T_{\|}} T_{\perp, \|}, \\
& p_{\perp, \|}^{\varphi}=\bar{n}\left(\frac{B}{B_{0}}\right)^{1-T_{\perp} / T_{\|}} T_{\perp, \|}\left(\exp \left\{\frac{\nu e \varphi}{T_{\|}}\right\}-1\right),
\end{aligned}
$$

To examine further the physics meaning of $p_{\perp, \|}^{\varphi}$, we note that in the case $\nu e \varphi / T_{\|} \ll 1$ one has

$$
p_{\perp, \|}^{\varphi} \approx \bar{n}\left(\frac{B}{B_{0}}\right)^{1-T_{\perp} / T_{\|}} \frac{T_{\perp, \|}}{T_{\|}} \nu e \varphi .
$$

One can see here that the electric potential gives rise to additional contribution to the pressure, which is what we refer to as the e-stress.

Note that the plasma temperature represents the total particle kinetic energy per unit volume. To estimate the ordering of e-stress one needs to estimate the total electrostatic potential energy due to the Coulomb interactions between non neutralized charged particle per unit volume. We use a circular column of length $L$ and cross section area $S$ to make this estimate. The e-stress ordering is then given as follows:

$$
\begin{aligned}
\nu n_{0} e \varphi & =\frac{1}{2 L S} \frac{1}{4 \pi \epsilon_{0}} \int_{-L / 2}^{L / 2} \int_{-L / 2}^{L / 2} \frac{\left(\nu n_{0} e\right)^{2}}{\left|x^{\prime}-x\right|} S^{2} d x d x^{\prime} \\
& \sim S \frac{\left(\nu n_{0} e\right)^{2}}{8 \pi \epsilon_{0}} \ln \left(\nu n_{0}\right)^{1 / 3}
\end{aligned}
$$

where the smallest distance between nonneutralized charged particles has been assumed to be $1 /\left(\nu n_{0}\right)^{1 / 3}$. This lower limit eliminates the singularity in this integration. One can also use the direct method to estimate the e-stress: i.e., the electric force per area exerted on one side of nonneutral tube by the charges on the other side. The result is equivalent to Eq. (7).

Using Eq. (7), one can estimate the ordering of the e-stress $\nu n_{0} e \varphi$. The density of nonneutralized electrons at tokamak edge $\nu n_{0}$ can be obtained by the Poisson equation with the experimentally observed radial electric field: $\nu n_{0}=\left(\epsilon_{0} / e\right)\left(d E_{r} / d r\right)$, where $\epsilon_{0}$ is vacuum permittivity, $r$ is minor radius, and $E_{r}(r)$ is the radial electric field. To explain the H-mode quiet feature in C-Mod experiments [8] as discussed in the introduction, we use the C-Mod H-mode discharge data as an example to analyze the orderings. From Ref. [12] one has 
the following parameter ranges: the radial electric field $E_{r} \sim 10^{5} \mathrm{~V} / \mathrm{m}$, the $E_{r}$ scale length $\Delta r \sim 10^{-2} \mathrm{~m}$, and pedestal density and temperature $n_{0} \sim 1.5 \times 10^{18} \mathrm{~m}^{-3}, T \sim 400 \mathrm{eV}$. Using these parameters one can find the ordering for nonneutralized electrons from the Poisson equation

$$
\nu n_{0} \sim \frac{8.85 \times 10^{-12}}{1.6 \times 10^{-19}} \frac{10^{5}}{10^{-2}} \sim 5.5 \times 10^{14}\left(1 / \mathrm{m}^{3}\right) .
$$

Finally, one obtains the following estimates: $\nu n_{0} e \varphi \sim 2.8 \times 10^{2}(\mathrm{~Pa})$, while the thermal pressure $p \sim 9.6 \times 10(\mathrm{~Pa})$. This indicates that for C-Mod the e-stress effect is significant. Noting further that the $E_{r}$ scale length is shorter than that of thermal pressure, one can conclude that the e-stress can be of the same order as thermal pressure. The e-stress effects can also be important to other tokamak discharges in certain parameter regimes [14, 15].

To further reduce Eq. (4) we note that the force $\nabla \cdot\left[p_{\perp}^{\varphi}(\mathcal{I}-\mathbf{b b})\right]$ in the perpendicular direction is similar to the radial electric force, which gives rise to the plasma rotation. Therefore, we can define

$$
\mathbf{u}_{\varphi}=-\frac{\mathbf{B} \times \nabla \cdot\left[p_{\perp}^{\varphi}(\mathcal{I}-\mathbf{b b})\right]}{\nu n_{0} e B^{2}} .
$$

Finally, equation (4) in the perpendicular direction reduces to

$$
\left.\rho \frac{d \mathbf{u}_{0}}{d t}\right|_{\perp}=-\nabla_{\perp} p_{\perp}^{T}-\frac{p_{\|}-p_{\perp}^{T}}{B} \mathbf{B} \cdot \nabla \mathbf{b}+\mathbf{j} \times \mathbf{B} .
$$

We can derive the radial equilibrium equation, i.e., the modified Grad-Shafranov equation [16], with the e-stress effects included

$$
R^{2} \nabla \cdot\left(\frac{\nabla \chi}{R^{2}}\right)=-\mu_{0} R^{2} \frac{\partial p_{\perp}^{T}}{\partial \chi}-g \frac{\partial g}{\partial \chi}-\mu_{0} \frac{R^{2} B}{B_{0}} \frac{\boldsymbol{\kappa} \cdot \nabla \chi}{|\nabla \chi|^{2}}\left(p_{\|}-p_{\perp}^{T}\right) .
$$

Here, $\chi$ is poloidal flux, $R$ denotes the major radius, $g(\chi) / R$ is the toroidal magnetic field, and $\boldsymbol{\kappa}=\mathbf{b} \cdot \nabla \mathbf{b}$. Note here that, since the pressure gradient is steep, the last term in Eq. (9) is small, as compared to the first term on the right, and therefore can be neglected in the pedestal region.

We can linearize Eq. (8) to obtain the generalized vorticity equation, by applying the operator $\nabla \cdot\left(\mathbf{B} / B^{2} \times \cdots\right)$ on it :

$$
\begin{aligned}
-\nabla \cdot\left[\frac{\mathbf{B}}{B^{2}} \times \delta\left(\rho \frac{d \mathbf{v}}{d t}\right)\right]= & \mathbf{B} \cdot \nabla \frac{\mathbf{B} \cdot \delta \mathbf{j}}{B^{2}}+\delta \mathbf{B} \cdot \nabla \sigma-\mathbf{j} \cdot \nabla \frac{\mathbf{B} \cdot \delta \mathbf{B}}{B^{2}} \\
& +\nabla \times \frac{\mathbf{B}}{B^{2}} \cdot \nabla \delta p_{\perp}^{T}+\frac{\mathbf{B} \times \boldsymbol{\kappa}}{B B_{0}} \cdot \nabla\left(\delta p_{\|}-\delta p_{\perp}^{T}\right),
\end{aligned}
$$


where the perturbed quantities are tagged with $\delta$. The forth term on the right is the thermal pressure driving term and the e-stress effect $\delta p_{\|}^{\varphi}$ resides in the last term. Note that $\nabla \times\left(\mathbf{B} / B^{2}\right) \sim(2 \mathbf{B} \times \boldsymbol{\kappa}) / B^{2}$. The similarity between the last two terms in Eq. (10) gives a general picture for e-stress effects as compared to the thermal pressure one.

To show the e-stress effects, let us next derive the generalized ballooning mode equation. Note that the radial electric field results in the $\mathbf{E} \times \mathbf{B}$ rotation and the rotation is assumed to be subsonic. We will consider the system stability in the local rotating frame. In this case the $\mathbf{E} \times \mathbf{B}$ rotation effect results only in the Doppler frequency shift: $\omega \rightarrow \hat{\omega} \equiv \omega+\operatorname{in} \Omega$ $[17,18]$, where $\omega$ is the mode frequency, $\Omega$ is the local rotation frequency, and $n$ is the toroidal mode number. For simplicity we consider the so-called $s$ - $\alpha$ equilibrium model [19]. For simplicity we ignore the so-called $\theta_{k}$ effects, including its rotation modification. In this model the ballooning mode equation is obtained from Eq. (10):

$$
\frac{d}{d \theta}\left(1+\Lambda^{2}\right) \frac{d \delta \varphi}{d \theta}+K \alpha_{c}(\cos \theta+\Lambda \sin \theta) \delta \varphi=0
$$

where $\Lambda=s \theta-\alpha_{c} \sin \theta, s$ is magnetic shear, $K=1+\left(\alpha_{\|}^{T}-\alpha_{c}\right) / 2 \alpha_{c}+\alpha_{\|}^{\varphi} / 2 \alpha_{c}, \alpha_{c}=$ $-2 \mu_{0}\left(R q^{2} / B^{2}\right)\left(d p_{\perp}^{T} / d r\right)$, and $\alpha_{\|}^{T, \varphi}=-2 \mu_{0}\left(R q^{2} / B^{2}\right)\left(d p_{\|}^{T, \varphi} / d r\right)$. Note that in the isotropic case $T_{\perp}=T_{\|}$, one has $\alpha_{\|}^{T}=\alpha_{c}$ and consequently the only factor affecting the parameter $K$ becomes the e-stress effect in $\alpha_{\|}^{\varphi}$. As discussed earlier the e-stress effects on the equilibrium can be ignored in the steep pressure gradient case. Therefore, only $\alpha_{c}$ affects the shear parameter $\Lambda$.

We use the ballooning mode equation, Eq. (11), to determine the stability boundaries, by invoking Newcomb's theorem No. 5 [20]. The conventional ballooning stability boundaries are recovered when $K=1$, which are plotted with solid curves in Fig. 2. Note that the e-stress increases from core plasma toward the edge, but the thermal pressure decreases radially. Therefore, in the pedestal region $\alpha_{\|}^{\varphi}$ and $\alpha_{c}$ have different signs, so that $K<1$. The stability boundaries for $K=0.8$ and 0.5 cases are plotted with dashed and dot-dashed curves respectively in Fig. 2. One can see that, when the e-stress effects are taken into account, the unstable parameter region shrinks. The pedestal region becomes more stable. In Fig. 2 we also plot the $K=1.5>1$ case (dotted curve), in which $\alpha_{\|}^{\varphi}$ and $\alpha_{c}$ have the same signs. In this case the first stability parameter region shrinks, while the second stability region disappears completely. As discussed earlier there is a jump of nonneutralized charge density across the last closed flux surface. Consequently, $\alpha_{\|}^{\varphi}$ becomes large and positive 
in this case. One can expect that the e-stress could contribute destabilizing effects across the last closed flux surface. From these analyses one can find that the e-stress effects are stabilizing inside the last closed flux surface, while destabilizing right across the last closed flux surface. This tendency is consistent with the building-up of steep pressure profile in the pedestal.

Note also that when the bootstrap current is taken into account the magnetic shear $s$ reduces considerably in a pedestal. The infernal modes tend to develop in this case [10]. From Fig. 2 one can see that the e-stress effects are most effective in the low shear region. This additional stability regime is particularly important to explain the infernal mode stability in the pedestal.

In conclusion, we point out that the nonneutralized electrons at tokamak edge contribute an electric compressive stress (i.e., e-stress) in the direction parallel to magnetic field. We have extended the CGL theory to include the compressive e-stress effect. In a strictly charge neutral plasma only thermal pressure is considered for the fluid cell interactions in the parallel direction. In a weakly nonneutral plasma, however, the fluid cell interaction is not only through the parallel thermal pressure, but also through the electric force. This results in what we call the e-stress. The existence of e-stress is simply because the nonneutralized electrons, confined in a compressed state by the closed filed lines, repulse each other. If the field lines holding nonneutralized elecrons were broken, the charged particles would be driven out due to the e-stress. The presence of the nonneutralized electrons at tokamak edge causes the equation of state to be changed. The potential energy from the electric repulsion, i.e., the e-stress, need to be included in the system in addition to the thermal energy. Because the density of nonneutralized charged particles increases from core toward plasma edge, the e-stress increases outwardly as well. This creates an additional magnetic well. Consequently, a stabilization effect in the pedestal region results. This linear stabilization regime helps to explain why some parameter regimes in the H-mode discharges can be rather quiet as observed experimentally.

This research is supported by Department of Energy Grants DE-FG02-04ER54742.

[1] F. Wagner, G. Becker, K. Behringer, et al., Phys. Rev. Lett. 49, 1408 (1982).

[2] R. D. Hazeltine, Phys. Fluids B1, 2031 (1989). 
[3] K. C. Shaing and E. C. Crume, Jr., Phys. Rev. Lett. 63, 2369 (1989).

[4] R. J. Groebner, Phys. Fluids B5, 2344 (1993).

[5] H. Biglari, P. H. Diamond, and P. W. Terry, Phys. Fluids B2, 1 (1990).

[6] K. C. Shaing, E. C. Crume, Jr., and W. A. Houlberg, Phys. Fluids B2, 1492 (1990).

[7] Linjin Zheng, "IOP Concise Physics: Advanced Tokamak Stability Theory" (Morgan \& Claypool Publishers, San Rafael, California, USA and IOP Publishing, Temple Circus, Bristol, UK, 2015).

[8] D. G. Whyte, A. E. Hubbard, J. W. Hughes, et al., Nucl, Fusion 50, 105005 (2010).

[9] C. E. Kessel, G. Giruzzi, A. C. C. Sips, et al, Nucl. Fusion 47, 1274 (2007).

[10] L. J. Zheng, M. T. Kotschenreuther, and P. Valanju, Nucl. Fusion 53, 063009 (2013).

[11] C. W. Horton, Jr., Phys. Fluids 12, 2132 (1969).

[12] R. M. McDermott, B. Lipschultz, J. W. Hughes, et al., Phys. Plasmas 16, 056103 (2009).

[13] G. F. Chew, M. L. Goldburger, and F. E. Low, Proc. R. Soc. London, Series A 236, 7 (1956).

[14] P. Gohil, K.H. Burrell and T.N. Carlstrom, Nucl, Fusion 38, 93 (1998).

[15] K. Crombe, Y. Andrew, T. M. Biewer, Plasma Phys. Control. Fusion 51, 055005 (2009).

[16] V. D. Shafranov, Reviews of Plasma Physics (Consultants Bureau, New York, 1966), vol. 2, p. 103.

[17] F. L. Waelbroeck and L. Chen, Phys. Fluids B3, 601 (1991).

[18] L.-J. Zheng, M. S. Chu, and L. Chen, Phys. Plasmas 6, 1217 (1999).

[19] J. W. Connor, R. J. Hastie, and J. B. Taylor, Phys. Rev. Lett. 40, 396 (1978).

[20] W. A. Newcomb, Ann. Phys. N. Y. 10, 232 (1960). 


\section{Figure captions:}

Fig. 1. Schematic explanation of charge density accumulation at tokamak edge. The small solid circles represent electrons. Both closed flux surfaces and scrape-off layer are given, together with the lower single-null divertor system.

Fig. 2. Stability boundaries for ballooning modes with e-stress taken into account in the $s$ $\alpha_{c}$ space. The $K=1$ case (solid curves) corresponds to the conventional ballooning stability boundaries with an isotropic pressure. The $K=0.5$ and 0.8 (dashed and dot-dashed curves) cases corresponds to the cases with $\alpha_{\|}^{\varphi}$ and $\alpha_{c}$ having different signs. E-stress is stabilizing in this case. The $K=1.5$ case corresponds to the case with $\alpha_{\|}^{\varphi}$ and $\alpha_{c}$ having the same signs (dotted curve). In the $K=1.5$ case the second stability regime disappears. E-stress is destabilizing in this case 


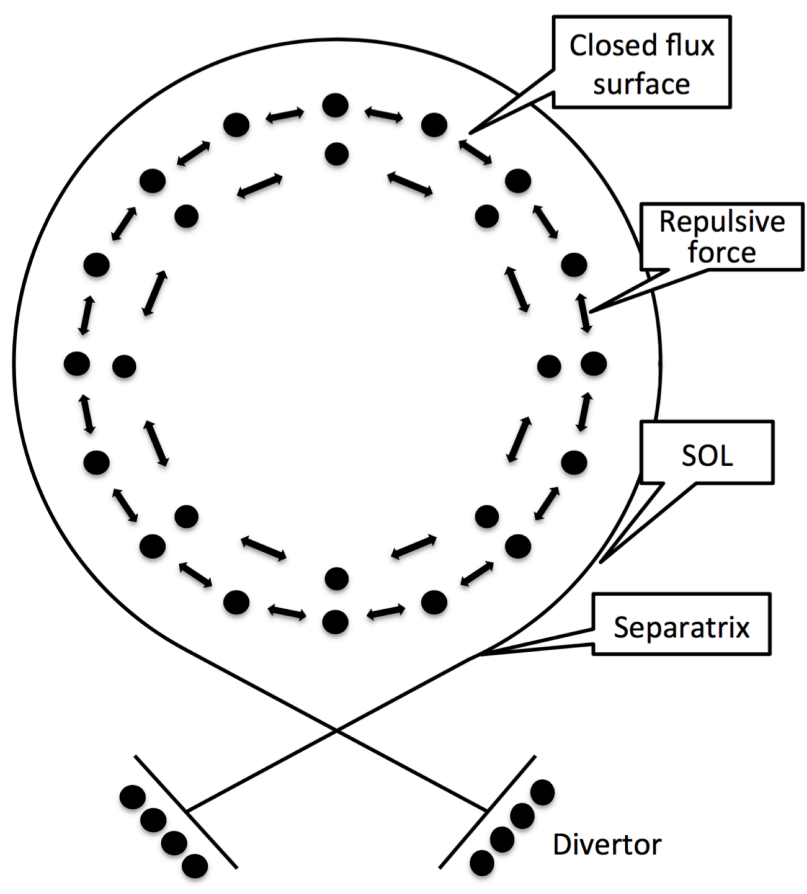

Fig. 1 


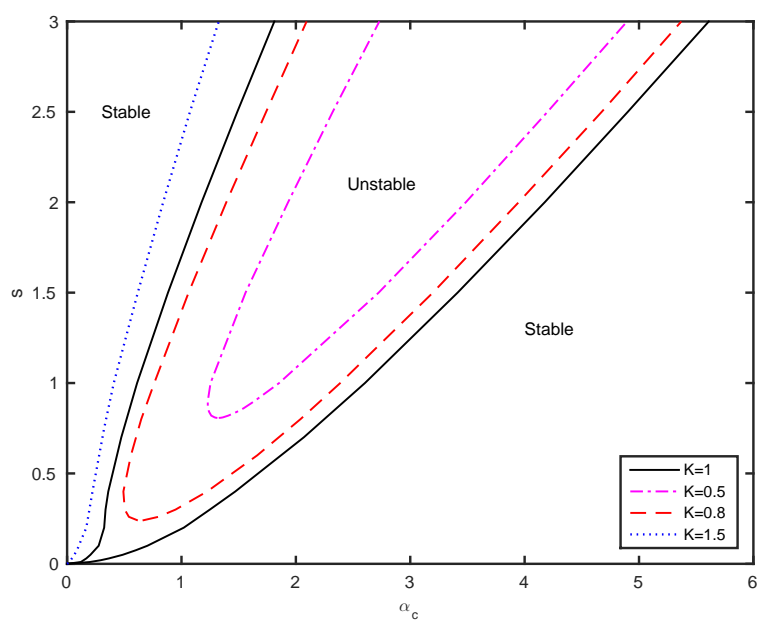

Fig. 2 